\title{
The responses and adaptations of microbial communities to salinity in farmland soils: A molecular ecological network analysis
}

\author{
Wei Zheng ${ }^{\mathrm{a}}$, Dongmei Xue ${ }^{\mathrm{a}, *}$, Xiangzhen $\mathrm{Li}^{\mathrm{b}}$, Ye Deng ${ }^{\mathrm{c}}$, Junpeng Rui ${ }^{\mathrm{b}}$, Kai Feng ${ }^{\mathrm{c}}$, \\ Zhong-liang Wang ${ }^{\mathrm{a}}$ \\ a Tianjin Key Laboratory of Water Resources and Environment, Tianjin Normal University, Tianjin 300387, China \\ ${ }^{\mathrm{b}}$ Key Laboratory of Environmental and Applied Microbiology, CAS, Environmental Microbiology Key Laboratory of Sichuan Province, Chengdu Institute of Biology, \\ Chinese Academy of Sciences, Chengdu 610041, China \\ c CAS Key Laboratory for Environmental Biotechnology, Research Center for Eco-Environmental Sciences, Chinese Academy of Sciences, Beijing 100085, China
}

\section{A R T I C L E I N F O}

\section{Keywords:}

Soil salinity

Microbial community structure

Molecular ecological network

Microbial interactions

\begin{abstract}
A B S T R A C T
Soil salinity is an increasing problem deteriorating soil fertility in degraded farmland soils. However, the responses and adaptations of microbial communities and interactions to salinity in farmland are not yet fully understood. In this study, we used 16S rRNA Miseq-sequencing technique to investigate the phylogenetic compositions, diversity and structure of soil microbial communities under different salinity conditions. The results indicated that prokaryotic diversity decreased with salinity. The change in prokaryotic community was primarily driven by salinity levels. The relative abundances of Proteobacteria, Bacteroidetes and Firmicutes were higher, and those of Acidobacteria and Thaumarchaeota were lower under high salinity than in medium and low salinity soils. Further, microbial network interactions changed along the salinity gradient revealed by a phylogenetic molecular ecological networks (pMENs) analysis. Salinity enhanced the interactions between microorganisms, evidenced by more links, higher average degree and average clustering coefficients within the pMENs in high salinity soils. Furthermore, we constructed the sub-networks of Flavobacterium and Acidobacteria_Gp4 to explore the changes of interactions among different microbial groups under salinity. We found that salinity shifted the interactions among different microbial taxa, and such changes vary among different microbial populations. This study provides solid evidences that microbial communities adapt to salinity through the adjustments of microbial compositions and interactions.
\end{abstract}

\section{Introduction}

Soil salinity is an increasingly serious problem in agricultural soils all over the world (Qadir et al., 2000). Many studies observe the influences of salinity on soil microbial communities (Pankhurst et al., 2001; Muhammad et al., 2006; Ghollarata et al., 2007; ValenzuelaEncinas et al., 2009; Johannes et al., 2011; Mavi et al., 2012; Campbell and Kirchman, 2013; Sun et al., 2015a,b). The effect of salt on soil microbes is stronger than that of heavy metals (Sardinha et al., 2003). However, it is still not fully conclusive on the salt effects on microbial community. The dominant parameters to characterize microbial responses to salt exposure were microbial biomass, respiration, microbial activity, composition, diversity and structure. For example, Muhammad et al. (2006) observe that the microbial biomass C decreased from approximately $190 \mu \mathrm{g} \mathrm{g}^{-1}$ to $80 \mu \mathrm{g} \mathrm{g}^{-1}$ in response to salinity stress ranged from 2.1 to $6.0 \mathrm{mg} \mathrm{g}^{-1}$. In contrast, Mavi and Marschner (2012) observed the microbial biomass C slightly increased from $93 \mu \mathrm{g} \mathrm{g}^{-1}$ to
$148 \mu \mathrm{g} \mathrm{g}^{-1}$ in response to salinity stress ranged from EC1.0 to EC2.5. Microbiological activities including soil respiration and enzyme activities are also depressed by salinity (Ghollarata and Raiesi, 2007). However, Rousk et al. (2011) reveal that soil salinity is not a decisive factor for bacterial growth.

The effects of salinity on microbial compositions and structure are widely studied (Pankhurst et al., 2001; Campbell and Kirchman, 2013). Sun et al. (2015a,b) report that high alkali-saline level could reduce soil microbial quantity, but not materially alter soil microbial community composition. The medium alkaline-saline soil had the highest diversity indices at the order and species level, in comparison with the high and low ones (Valenzuela-Encinas et al., 2009). Killham (1994) describes two main adaptation strategies of microorganisms to osmotic stress (e.g. salinity, drought or freezing), including ion accumulation in the cell to exclude salt solute, and production of organic acids to antagonize salt gradient. However, these mechanisms are known from single microorganisms, but seldom focus on microbial interactions among

\footnotetext{
* Corresponding author.

E-mail address: xuedongmeixdm@126.com (D. Xue).
} 
various populations. We are still not fully understood how a community adapts to salinity through community-level adjustments of the compositions and interactions.

Microbial interactions may provide system-level adaptations of prokaryotic communities to soil salinity. Co-occurrence of prokaryotic populations in a community reflects their similar niche adaptations of the co-occurring species, or interspecies interactions, either by competition or by cooperation (Rui et al., 2015). It is not clear how the salinity drives the changes of microbial interactions. Many network methods have been developed, including equation-based network methods (Yeung et al., 2002; Gardner et al., 2003), Bayesian network methods (Gerstung et al., 2009) and relevance/co-expression network methods (Zhang and Horvath, 2005; Horvath et al., 2006; Oldham et al., 2006). However, most studies use arbitrary thresholds, and thus the constructed networks are subjective rather than objective (Barabasi and Oltvai, 2004). The phylogenetic molecular ecological networks (pMENs) have been proposed based on a novel conceptual framework using a random matrix theory (RMT)-based approach (Deng et al., 2012). It provides good solutions to some common problems concerning high-throughput metagenomic data (Deng et al., 2012). The pMENs has been applied to characterize network interactions of microbial communities in response to elevated $\mathrm{CO}_{2}$ (Zhou et al., 2011), heavy metal pollutions (Yin et al., 2015), ocean acidification (Wang et al., 2015) and livestock grazing (Sun et al., 2015a,b).

Different soil salinity gradients have been formed along the Bohai Bay in Tianjin, China, due to interlace of fluvial outwash and marine deposit, shallow groundwater level and unreasonable exploration of groundwater resources (Wang et al., 2011). High soil salinity seriously influences the crop production and sustainable development of agriculture in Tianjin. In this study, the pMENs was applied to investigate the responses of soil microbial communities and microbial interactions to salinity changes in Tianjin farmland soils in May and November. We hypothesized that (1) the phylogenetic diversity and structure of microbial community would shift under different salinity conditions; (2) soil salinity would affect microbial network interactions among different salinity gradients.

\section{Material and methods}

\subsection{Study sites and sampling}

Field sites located in the Bohai Bay in Tianjin municipality. The research area is affected by the warm temperate semi-humid continental monsoon climate with an average annual temperature of $11.4-12.9^{\circ} \mathrm{C}$. The annual precipitation is $520-660 \mathrm{~mm}$, with $75 \%$ of the total precipitation occurring from June to August (Yue et al., 2010). Due to regional differences in topography, precipitation, evaporation, groundwater depth and soil properties, soil salinity gradients are formed from north to south in Tianjin. Six farmland sites were selected along the salinity gradients, including low salinity region (L group) with braunerde soil (L1: $40^{\circ} 04^{\prime} 14.87^{\prime \prime} \mathrm{N}, \quad 117^{\circ} 20^{\prime} 01.90^{\prime \prime} \mathrm{E}$; L2: $40^{\circ} 05^{\prime} 25.91^{\prime \prime} \mathrm{N}, 117^{\circ} 38^{\prime} 10.21^{\prime \prime} \mathrm{E}$ ); medium salinity region (M group) with fluvo-aquic soil (M1: $39^{\circ} 36^{\prime} 54.49^{\prime \prime} \mathrm{N}, 116^{\circ} 58^{\prime} 04.20^{\prime \prime} \mathrm{E}$; M2: $39^{\circ} 32^{\prime} 09.31^{\prime \prime} \mathrm{N}, 116^{\circ} 59^{\prime} 32.31^{\prime \prime} \mathrm{E}$ ), and high salinity region (H group) with coastal saline soil (H1: $38^{\circ} 43^{\prime} 09.78^{\prime \prime} \mathrm{N}, 117^{\circ} 26^{\prime} 44.00^{\prime \prime} \mathrm{E}$; $\left.\mathrm{H} 2: 38^{\circ} 49^{\prime} 08.84^{\prime \prime} \mathrm{N}, 117^{\circ} 03^{\prime} 38.57^{\prime \prime} \mathrm{E}\right)$. The physico-chemical properties in 6 sampling sites were shown in Table S1. The salt levels in six sampling sites at different layers were shown in Table S2. The hierarchical cluster of soil sampling sites based on soil salinity was exhibited in Fig. S1.

Samplings were conducted twice in May and November 2013. The unsaturated zone cores were collected with a $5.5 \mathrm{~cm}$ diameter hollowstem hand auger. Samples were taken continuously in total 10 layers from the ground surface to a depth of $3 \mathrm{~m}$ in a profile, including: $0-0.2 \mathrm{~m}, 0.2-0.4 \mathrm{~m}, 0.4-0.6 \mathrm{~m}, 0.6-0.8 \mathrm{~m}, 0.8-1.0 \mathrm{~m}, 1.0-1.3 \mathrm{~m}$, $1.3-1.6 \mathrm{~m}, 1.6-2.0 \mathrm{~m}, 2.0-2.5 \mathrm{~m}$ and $2.5-3.0 \mathrm{~m}$. Samples were stored in polyethylene bags. Moisture content was determined by drying a minimum of $50 \mathrm{~g}$ of soil sample at $108{ }^{\circ} \mathrm{C}$ for $24 \mathrm{~h}$. Soil water extracts were obtained by adding $200 \mathrm{ml}$ Mili Q water to $100 \mathrm{~g}$ soil sample, shaking $45 \mathrm{~min}$ at room temperature, centrifuging at $4000 \mathrm{rpm}$, and filtering supernatant. Soil water extracts were used to measure $\mathrm{pH}$ and salinity by a portable analyzer (Orion Star A329, Thermo, USA), $\mathrm{SO}_{4}{ }^{2-}$ and $\mathrm{Cl}^{-}$by an ion chromatography (ICS-2100, Dionex, USA), $\mathrm{K}^{+}, \mathrm{Na}^{+}$, $\mathrm{Ca}^{2+}, \mathrm{Mg}^{2+}$ by a Inductively coupled plasma atomic emission spectrometry (Optima 8300, PE, USA), TOC and TN by a total organic carbon analyzer (vario, Elementar, Germany); and $\mathrm{NO}_{3}{ }^{-}$and $\mathrm{NH}_{4}{ }^{+}$by a continuous flow analyzer (Auto Analyzer 3, Seal, Germany). Part of soil samples were freeze dried and stored at $-20{ }^{\circ} \mathrm{C}$ for genomic DNA extraction.

\subsection{DNA extraction, miseq sequencing and data analysis}

DNA extraction was extracted using Ezup genomic DNA extraction kit for soil (Sangon Biotech, China, Cat\# SK8264). DNA concentration and quality were checked using a NanoDrop Spectrophotometer. Extracted DNA was diluted to $10 \mathrm{ng} \mathrm{LL}^{-1}$.

Universal primer 515F (5'-GTGCCAGCMGCCGCGGTAA- $3^{\prime}$ ) and 806R (5'-GGACTACHVGGGTWTCTAAT-3') was used to amplify the V4 hypervariable region of $16 \mathrm{~S}$ rRNA gene for pyrosequencing using Miseq sequencer (Lin et al., 2016). The PCR mixture (25 $\mu$ l) contained 1 x PCR buffer, $1.5 \mathrm{mM} \mathrm{MgCl}_{2}$, each deoxynucleoside triphosphate at $0.4 \mathrm{mM}$, each primer at $1.0 \mu \mathrm{M}$ and $0.5 \mathrm{U}$ of Ex Taq (TaKaRa, Dalian) and $10 \mathrm{ng}$ soil genomic DNA. The details about PCR amplification program were described before (Li et al., 2014). In total, 115 samples were sequenced using Reagent Kit v2 $2 \times 250$ bp by the Illumina Miseq platform at Environmental Genome Platform of Chengdu Institute of Biology.

The sequence data were processed using QIIME Pipeline-Version 1.7.0 (http://qiime.org/). All sequence reads were trimmed based on their unique barcodes. The sequences with high quality (length $>300$ bp, without ambiguous base ' $N$ ', and average base quality score $>20$ ) were used for downstream analysis. Chimera sequences were removed using the UCHIME algorithm (Edgar et al., 2011). Sequences were clustered by the complete-linkage clustering method incorporated in the RDP pyro pipeline. Operational taxonomic units (OTUs) were classified using a $97 \%$ nucleotide sequence similarity cutoff. Taxonomy was assigned using the Ribosomal Database Project classifier (Wang et al., 2007). All the samples were randomly-resampled to 2590 reads. The indices of alpha-diversity were calculated, including chao1 estimator of richness, observed species and Shannon's diversity. The original sequence data were deposited at the European Nucleotide Archive by accession PRJEB21751 (http://www.ebi.ac.uk/ena/data/view/ PRJEB21751).

\subsection{Statistical analysis}

The hierarchical cluster analysis of sampling sites based on soil salinity was performed by IBM SPSS 21 . The average rarefaction curves among $\mathrm{H}, \mathrm{M}$ and L groups were generated from the observed species. The univariate ANOVA was used to identify the factors making the differences of microbial community diversity by IBM SPSS 21 . Then, the one-way ANOVA based on Chao1 richness and Shannon diversity index was applied to compare microbial community diversity by IBM SPSS 21. The principal coordinate analysis (PCoA) (Wang et al., 2015) was used to compare the microbial community structure of six sampling sites by CANOCO 5.0 based on Bray-Curtis distance using the relative abundance data of OTU. The ANOSIM of microbial composition was applied to test the differences among three groups by Primer 7. The Mantel test (Yin et al., 2015) was applied to evaluate the correlations between microbial communities with environmental variables using PCORD 5.0. The principal coordinate analysis was performed with averaging values from the ten layers of each point, and all other analyses were performed with raw data. 


\section{4. pMENs construction and network analysis}

Phylogenetic molecular ecological networks (pMENs) analysis was performed based on the relative abundances of all samples through the pMENs analysis pipeline (http://ieg2.ou.edu/MENA; Zhou et al., 2010, 2011; Deng et al., 2012). The whole process and details are given in a previous MENA study elsewhere (Deng et al., 2012). Briefly, OTUs in at least 9 out of each group were used to construct network. Various network properties such as average degree, average path distance, average clustering coefficient and modularity index were characterized based on similarity matrices. The network modules were generated by fast greedy modularity optimization (Newman, 2006). Identification of key module members was based on within-module connectivity (Zi) and among-module connectivity (Pi) of each node (Olesen et al., 2007). Besides, based on singular value decomposition (SVD), eigengene network analysis was performed to summarize the gene abundance data from each module in pMENs. Finally, mantel tests were used to measure the correlations between pMENs and environmental properties. The Cytoscape 3.1.1 was used to visualize network graphs. Since we were primarily interested in the impact of seasonal salinity on network interactions, the pMENs were constructed under $\mathrm{H}, \mathrm{M}$ and L groups for May and November, respectively.

\section{Results}

\subsection{Phylogenetic compositions and structure of soil microbial communities}

A total of 1199572 high quality 16S rRNA gene sequences were obtained for all 115 samples (Table S3). They were resampled to 2590 sequences per sample, which were clustered into 7403 OTUs. The rarefaction curves showed that our sequencing efforts were enough to distinguish the differences between different salinity levels (Fig. S2). The univariate ANOVA results showed that the effects of soil depth on prokaryotic community diversity were not significant, but those of salinity were significant. Therefore, the key factor causing the differences of community diversity was not soil depth, but salinity (Table S4). Both the Shannon and Chao1 indices demonstrated significant difference between the $H$ group and L group $(P<0.05)$ in May, while only the Shannon index showed a significant difference between the $\mathrm{H}$ and $\mathrm{L}$ groups $(\mathrm{P}<0.05)$ in November (Table 1$)$.

Furthermore, the PCoA results further revealed that the microbial community structures were changed by salinity in both samplings (Fig. 1). Similar community compositions at phylum level were observed in May and November samples at the same salinity level (Fig. 2). The ANOSIM showed that microbial compositions were significantly different among $\mathrm{H}, \mathrm{M}$ and $\mathrm{L}$ group at both samplings (Table 2). The Mantel tests indicated that the shifts in microbial communities were significantly correlated with salinity, $\mathrm{K}^{+}, \mathrm{Na}^{+}, \mathrm{Mg}^{2+}, \mathrm{NO}_{3}{ }^{-}, \mathrm{Cl}^{-}$and $\mathrm{SO}_{4}{ }^{2-}(\mathrm{P}<0.05)$ both samplings (Table 3$) . \mathrm{Ca}^{2+}, \mathrm{TN}$, moisture and $\mathrm{pH}$ were significantly correlated with microbial communities either in May or November.

The top seven phyla were Proteobacteria, Thaumarchaeota, Acidobacteria, Bacteroidetes, Actinobacteria, Chloroflexi and Firmicutes among $\mathrm{H}, \mathrm{M}$ and $\mathrm{L}$ groups in both samplings. These phyla accounted for more than $70 \%$ of the total sequences at all sites (Fig. 2). However, the relative abundances of Proteobacteria, Bacteroidetes and Firmicutes were apparently higher in $\mathrm{H}$ group than those in $\mathrm{M}$ and $\mathrm{L}$ groups both samplings. In contrast, the relative abundances of Thaumarchaeota and Acidobacteria were relatively lower in $\mathrm{H}$ group compared with those in $\mathrm{M}$ and $\mathrm{L}$ groups. In May, the most abundant genera were Nitrososphaera (6.46\%) and Lactobacillus (3.31\%) in H group, Nitrososphaera (16.43\%) and Nitrosopumilus (13.09\%) in M group, and Nitrososphaera (18.22\%) and Gp4 (6.41\%) in L group. In November, the most abundant genera were Bacteroides $(6.21 \%)$ and Lactobacillus $(3.60 \%)$ in $\mathrm{H}$ group, $\mathrm{Ni}$ trososphaera (9.62\%) and Bacteroides (5.05\%) in M group, and Nitrososphaera (12.91\%) and Gp4 (6.83\%) in L group (Table S5).

\subsection{Microbial community interactions}

To understand the interactions of microbial populations in $\mathrm{H}, \mathrm{M}$ and $\mathrm{L}$ microbial communities, we used OTU data of $16 \mathrm{~S}$ rRNA sequences to construct pMENs for $\mathrm{H}, \mathrm{M}$ and L groups by RMT-based network approach. Major topological properties of six empirical MENs (H-MEN, MMEN, and L-MEN in May; H-MEN, M-MEN, and L-MEN in November) of microbial communities showed that salinity did influence the connectivity of micro-organisms with similar threshold $(0.710$ for $\mathrm{H}, 0.730$ for $\mathrm{M}$ and 0.730 for $\mathrm{L}$ in May, and 0.710 for $\mathrm{H}, 0.720$ for $\mathrm{M}$ and 0.710 for $\mathrm{L}$ in November, respectively) in two sampling times (Table 4). Compared with M- and L-MENs, the H-MENs demonstrated less total nodes (259 and 262), less modules (17 and 15), but more links (912 and 1259), higher average degree (7.042 and 9.611) and average clustering coefficients (0.560 and 0.587) both in May and November. This indicated that salinity may enhance the interaction between microorganisms, and the network became denser with high salinity in May and November, respectively. The higher modularity suggested that the microbial communities were highly complex (Olesen et al., 2007). It was interesting to see that the modularity demonstrated highest value (0.862) in May while the lowest value $(0.696)$ in November for $\mathrm{H}$ MENs. The values of $R$ square of power-law were above 0.7 , and this indicated ecological networks based on RMT should be scale-free (Zhou et al., 2010). The average path distance (GD) means the shortest path between two nodes (Wang et al., 2015). It demonstrated irregular variation based on salinity gradients.

Strong positive correlations were observed in all six pMENs, while negative correlations were rare (Fig. S3). It implied that microbes might cooperate more to adapt to high salinity or similar niches. Further, we constructed the sub-networks of Flavobacterium and Acidobacteria_Gp4 to explore the possible interactions between Flavobacterium/ Acidobacteria_Gp4 and other microbes. The top three Flavobacterium OTUs in $\mathrm{H}$ group had more complex interactions than their corresponding OTUs in $\mathrm{M}$ and $\mathrm{L}$ groups, evidenced by more nodes and links (Fig. 3), while Acidobacteria_Gp4 had much less complex interactions in H group (Fig. 4). For example, in November samplings, OTU383 was connected with ten nodes in L-MEN and three nodes in M-MEN, but only one in H-MEN. It might implicate that the interactions of Acidobacteria_Gp4 with other microbes were weakened at high salinity environments, evidenced by less nodes and links.

Furthermore, eigengene analysis (Langfelder and Horvath, 2007;

Table 1

Shannon and Chao1 indices in May and November samples.

\begin{tabular}{|c|c|c|c|c|}
\hline \multirow[t]{2}{*}{ Group } & \multicolumn{2}{|l|}{ May } & \multicolumn{2}{|l|}{ November } \\
\hline & Shannon & Chao1 & Shannon & Chao1 \\
\hline $\mathrm{H}$ & $7.63 \pm 0.65^{\mathrm{a}}$ & $958.22 \pm 195.69^{\mathrm{a}}$ & $7.13 \pm 1.07^{\mathrm{a}}$ & $951.99 \pm 166.42^{\mathrm{a}}$ \\
\hline M & $7.89 \pm 0.46^{\mathrm{ab}}$ & $1232.50 \pm 120.85^{\mathrm{b}}$ & $7.78 \pm 0.52^{\mathrm{ab}}$ & $1055.01 \pm 99.30^{\mathrm{a}}$ \\
\hline $\mathrm{L}$ & $8.10 \pm 0.34^{\mathrm{b}}$ & $1245.13 \pm 113.05^{\mathrm{b}}$ & $7.85 \pm 0.37^{\mathrm{b}}$ & $1003.14 \pm 122.22^{\mathrm{a}}$ \\
\hline
\end{tabular}

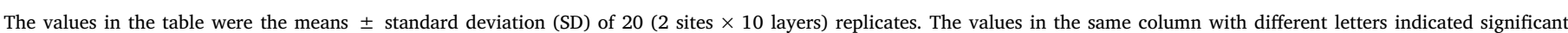
differences (at $\mathrm{P}<0.05$ ). 

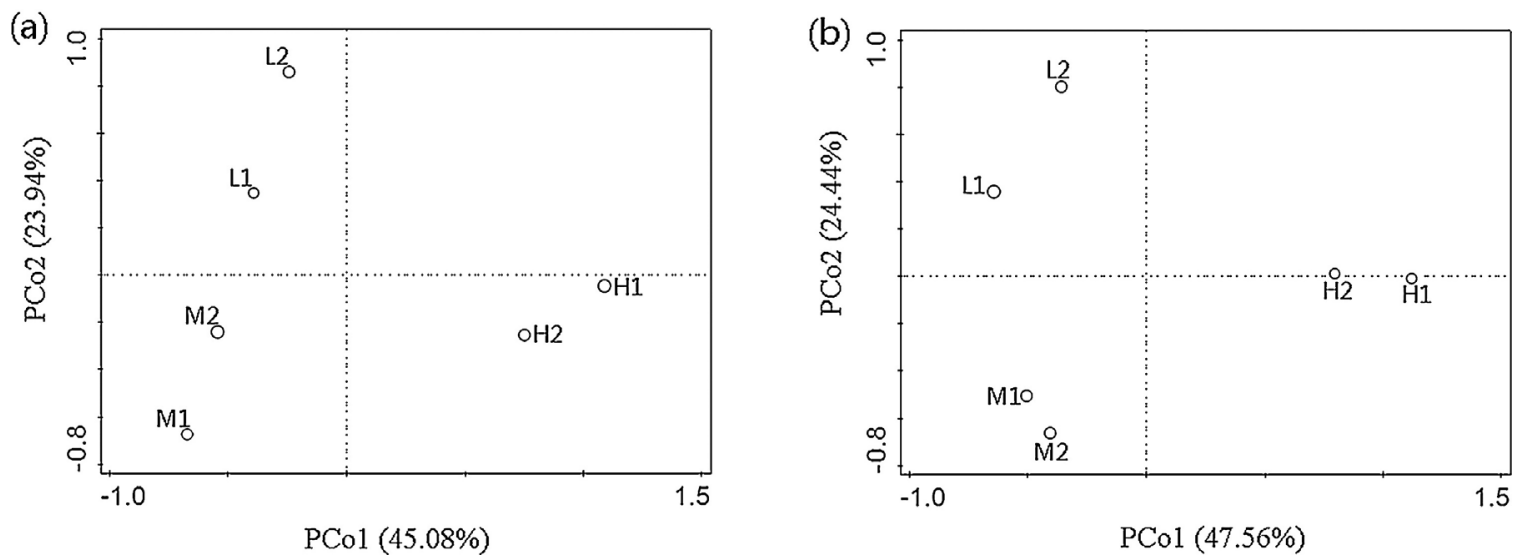

Fig. 1. Principle coordination analysis of microbial communities from six sampling sites based on the OTU relative abundances in May (a) and November (b).

Horvath and Dong, 2008) was performed to reveal the higher-order organization of the constructed pMENs. In this analysis, each module is represented by its singular value decomposition (SVD) of abundance profile, which is referred to as the module eigengene. The results showed that module eigengene explained $43-83 \%$ of the variances in relative OTU abundances across different samples in $\mathrm{H}$ group, 34-66\% in $\mathrm{M}$ group and $38-75 \%$ in $\mathrm{L}$ group in May, respectively. In November, module eigengene explained $41-94 \%$ of the variances in $\mathrm{H}$ group, $38-70 \%$ in $\mathrm{M}$ group and $36-90 \%$ in L group, respectively. For example, in May, module E10 in $\mathrm{H}$ group had 31 OTUs derived from Bacteroidetes, Chloroflexi, Proteobacteria, Actinobacteria, Euryarchaeota and Deinococcus-Thermus. Its eigengene could explain $48 \%$ of all variations (Fig. S4). In addition, the relationships between microbial network modules and environmental properties were analyzed with Mantel tests. It was found that $\mathrm{Na}^{+}, \mathrm{Mg}^{2+}, \mathrm{Cl}^{-}, \mathrm{SO}_{4}{ }^{2-}$ and salinity were significantly correlated with module E10 in $\mathrm{H}$ group.

Connectivity analysis among or within the modules showed that different OTUs (nodes) played distinct roles in the pMENs (Fig. 5). From an ecological perspective, the peripherals may represent specialists, whereas module hubs and connectors may be more generalists and network hubs may be super-generalists (Olesen et al., 2007; Deng et al., 2012). Two connectors (OTU2269 and OTU968) were observed in $\mathrm{H}$ group network, which were derived from Firmicutes (May) and Bacteroidetes (November). One module hub (OTU70) was detected in the M group network, which was derived from Actinobacteria (November). One connector and four module hubs were present in the L group network, which included Acidobacteria OTU59 (November), Thaumarchaeota OTU157 (May), Proteobacteria OTU350 (May), Actinobacteria OTU79 (November) and Unclassified OTU578 (May). No network hubs were detected in any pMENs. The above results suggested that salinity markedly altered the network structure and topological roles of individual OTUs and key microbial populations.
Table 2

ANOSIM of microbial compositions between three groups in May and November.

\begin{tabular}{llllll}
\hline Groups compared & \multicolumn{2}{l}{ May } & & & \multicolumn{2}{l}{ November } & \\
\cline { 2 - 3 } \cline { 5 - 6 } & $\mathrm{R}$ & $\mathrm{P}$ & & $\mathrm{R}$ & $\mathrm{P}$ \\
\hline H-M-L group & 0.490 & 0.001 & & 0.329 & 0.001 \\
\hline
\end{tabular}

$\mathrm{R}>0$ indicated that differences between three groups were greater than within-groups.

Table 3

The correlations between environmental variables and microbial community composition in May and November by Mantel test.

\begin{tabular}{lll}
\hline Environmental Variable & May & November \\
\hline $\mathrm{K}^{+}$ & $0.1873^{\text {*** }}$ & $0.1808^{\text {*** }}$ \\
$\mathrm{Ca}^{2+}$ & 0.1174 & $0.2143^{* * *}$ \\
$\mathrm{Na}^{+}$ & $0.3363^{\text {*** }}$ & $0.2569^{* *}$ \\
$\mathrm{Mg}^{2+}$ & $0.2247^{\text {*** }}$ & $0.1538^{*}$ \\
$\mathrm{NH}_{4}{ }^{+}$ & 0.0249 & -0.0588 \\
$\mathrm{NO}_{2}{ }^{-}$ & -0.0547 & -0.0209 \\
$\mathrm{NO}_{3}{ }^{-}$ & $0.1799^{* * *}$ & $0.3520^{* * *}$ \\
$\mathrm{Cl}^{-}$ & $0.3521^{* * *}$ & $0.2270^{* * *}$ \\
$\mathrm{SO}_{4}{ }^{2-}$ & $0.3277^{* * *}$ & $0.2949^{* * *}$ \\
$\mathrm{TOC}^{\mathrm{TN}}$ & 0.0149 & 0.0097 \\
$\mathrm{moisture}$ & 0.0117 & $0.3405^{* *}$ \\
$\mathrm{pH}$ & $0.1223^{* *}$ & 0.0902 \\
salinity & 0.0444 & $0.1571^{* *}$ \\
\hline
\end{tabular}

Significanc:

$* \mathrm{P}<0.05$

$* * \mathrm{P}<0.01$.
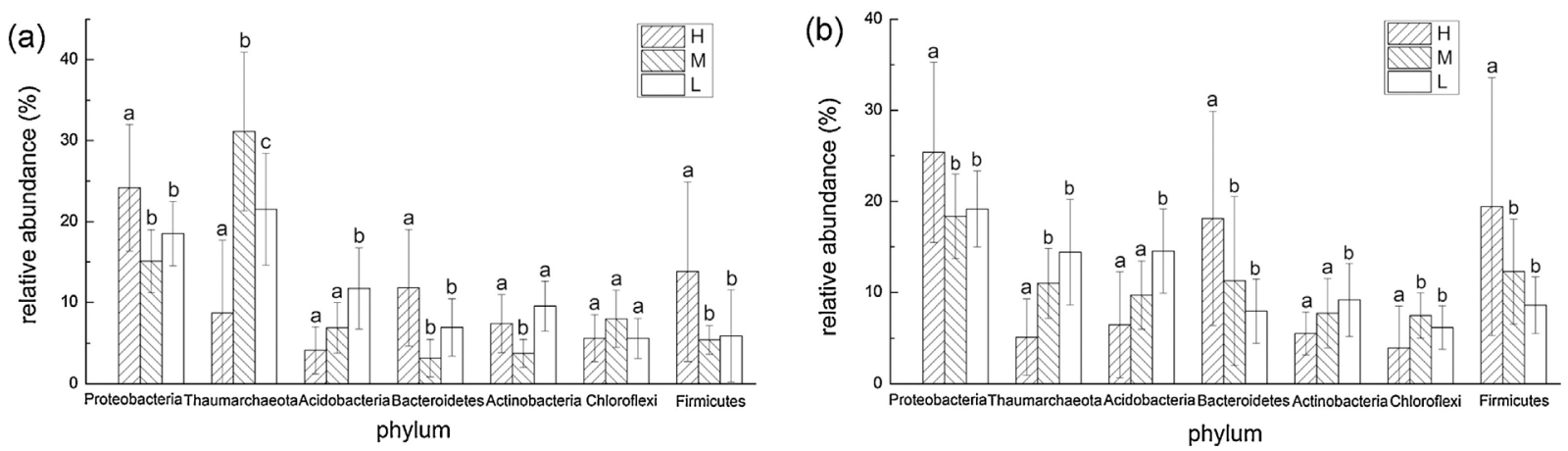

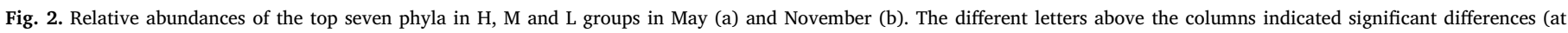
$\mathrm{P}<0.05)$ among three groups. The error bar indicated standard deviation (SD) of 20 (2 sites $\times 10$ layers $)$ replicates. 
Table 4

Topological properties of molecular ecological networks under different salinities.

\begin{tabular}{|c|c|c|c|c|c|c|c|c|c|c|c|}
\hline \multicolumn{2}{|l|}{ Community } & \multirow{2}{*}{$\begin{array}{l}\text { No. of } \\
\text { original OTUs } \\
2713\end{array}$} & \multirow{2}{*}{$\begin{array}{l}\text { Similarity } \\
\text { threshold }\end{array}$} & \multirow{2}{*}{$\begin{array}{c}\text { Total } \\
\text { nodes } \\
259\end{array}$} & \multirow{2}{*}{$\begin{array}{l}\text { Total links } \\
912\end{array}$} & \multirow{2}{*}{$\begin{array}{l}\begin{array}{l}\text { R square of } \\
\text { power-law }\end{array} \\
0.777\end{array}$} & \multirow{2}{*}{$\begin{array}{l}\begin{array}{l}\text { Average } \\
\text { degree } \\
\text { (avgKK) }\end{array} \\
7.042\end{array}$} & \multirow{2}{*}{$\begin{array}{l}\text { Average clustering } \\
\text { coefficient (avgCC) }\end{array}$} & \multirow{2}{*}{$\begin{array}{l}\text { Average path } \\
\text { distance (GD) } \\
5.327\end{array}$} & \multirow{2}{*}{$\begin{array}{l}\text { Module } \\
17\end{array}$} & \multirow{2}{*}{$\begin{array}{l}\text { Modularity } \\
0.862\end{array}$} \\
\hline May & $\mathrm{H}$ group & & & & & & & & & & \\
\hline & M group & 2912 & 0.730 & 275 & 725 & 0.840 & 5.273 & 0.369 & 3.048 & 25 & 0.691 \\
\hline & L group & 2690 & 0.730 & 349 & 880 & 0.696 & 5.043 & 0.434 & 7.799 & 25 & 0.860 \\
\hline \multirow[t]{3}{*}{ November } & $\mathrm{H}$ group & 2379 & 0.710 & 262 & 1259 & 0.604 & 9.611 & 0.587 & 4.750 & 15 & 0.696 \\
\hline & M group & 2252 & 0.720 & 281 & 929 & 0.803 & 6.612 & 0.410 & 5.748 & 18 & 0.782 \\
\hline & L group & 2243 & 0.710 & 267 & 657 & 0.815 & 4.921 & 0.395 & 4.021 & 22 & 0.834 \\
\hline
\end{tabular}

In addition, we explored the correlations between environmental variables and pMENs microbial compositions (Table S6). Thaumarchaeota, Bacteroidetes and Proteobacteria were significantly correlated with environmental properties under $\mathrm{H}$ group in May and November; Bacteroidetes and Actinobacteria were significant under M group in May, and Actinobacteria were significant under L group in November. The results suggested that the network interactions among different phylogenetic groups/populations were dramatically shifted by salinity, and such impacts in network interactions were significantly correlated to soil environmental properties.

\section{Discussions}

Understanding the shift of microbial composition and structure under different soil salinity gradients is critical to reveal the adaptation mechanisms of microbial community to salinity. In this study, significant differences in the microbial community compositions and microbial interactions networks were observed along salinity gradients. Salinity is identified to be the key factor driving the variations of prokaryote community diversity, structure and microbial interactions. Our study provided deep insights into the responses and adaptations of soil prokaryotic community to salinity at community level based on microbial interactions analysis.

More Proteobacteria, Firmicutes and Bacteroidetes, but less Thaumarchaeota and Acidobacteria were observed in $\mathrm{H}$ group compared to $\mathrm{M}$ and L groups. Thus, the phyla Proteobacteria, Firmicutes and Bacteroidetes are likely more resistant to high salinity, while Acidobacteria and Thaumarchaeota are less resistant or susceptible to high salinity. In a previous study, Sand et al. (2014) demonstrate that Acinetobacter baylyiADP1 (Proteobacteria) can cope with high salinity by uptake and accumulation of the well-known compatible solute glycine betaine. Another study (Samaei et al., 2013) shows that some
Proteobacteria such as Acinetobacter radioresistens and Pseudomonas aeruginosa (strain 1) are halotolerant, and another Pseudomonas aeruginosa strain 2 may be halophile. Similarly, Egamberdieva et al. (2008) also report that two Acinetobacter strains and Pseudomonas aeruginosa are salt tolerant. Above results and our data supported that Proteobacteria is likely resistant to high salinity.

A recent study (Hidri et al., 2016) in saline soil demonstrates that Bacillus sp. (Firmicutes) has a great surviving capacity under salt conditions. Halobacillus salinus (Firmicutes) and Bacillus simplex (Firmicutes) are the bacteria suitable for bioremediation in hypersaline conditions (Nicholson and Fathepure, 2005). These results supported that Firmicutes possess the high salinity resistance. Some studies also show that Bacteroidetes is a phylum resistant to salt (Valenzuela-Encinas et al., 2009; Keshri et al., 2013), and it can be one of dominant populations in alkaline saline soil (Valenzuela-Encinas et al., 2009). A similar study (Keshri et al., 2013) shows that the dominant phylum in the haloalkaline soil is Bacteroidetes followed by Proteobacteria.

On the contrary, microbes with low resistance to high salinity would decrease their abundances in $\mathrm{H}$ group samples, such as Acidobacteria and Thaumarchaeota. Acidobacteria was are reported to be abundant in medium-saline soils (Valenzuela-Encinas et al., 2009), saline sediments from Qinghai Lake (Dong et al., 2006) and mangrove sediments of Sundarban (Ghosh et al., 2010). However, higher percentages of Acidobacteria are also observed in low salinity soil than that in high salinity soil (Yang et al., 2016), which is consistent with our study. Archaeal phylum Thaumarchaeota are chemolithoautotrophic ammonia-oxidizers including genus Nitrososphaera. Our data showed that the Nitrososphaera genus was abundant in most samples and decreased in $\mathrm{H}$ salinity, indicating their sensitivities to salinity. These Thaumarchaeota microbes play important roles in carbon and nitrogen cycles. Thus, changes in salinity may significantly alter biogeochemical cycles in farmland soil. (a)

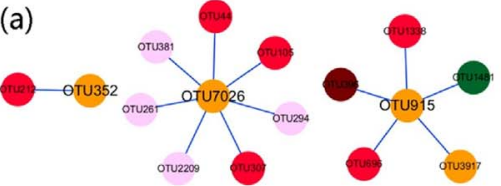

(d)
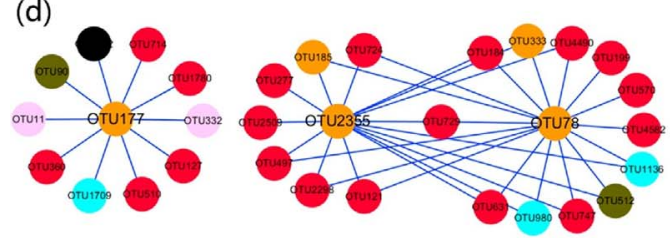

(b)

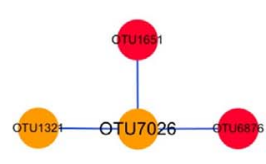

(e)
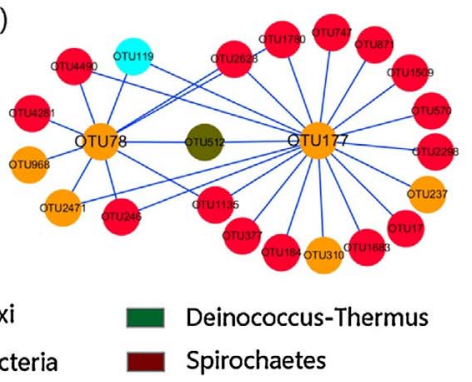

Deinococcus-Thermus

Spirochaetes

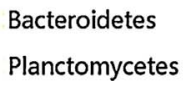

Chloroflexi

Proteobacteria (c)

(f)
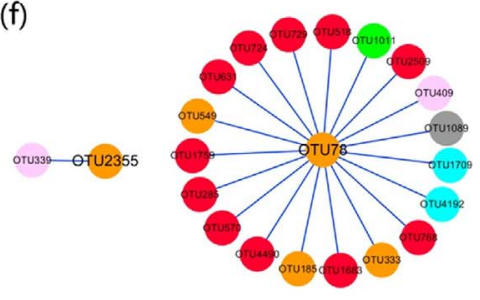

Firmicutes

Unclassified

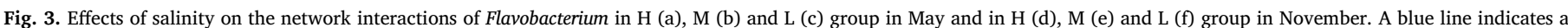

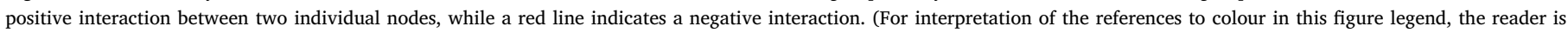
referred to the web version of this article.) 
(a)

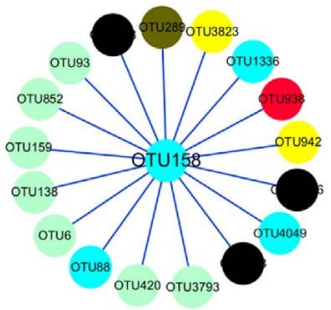

(d)
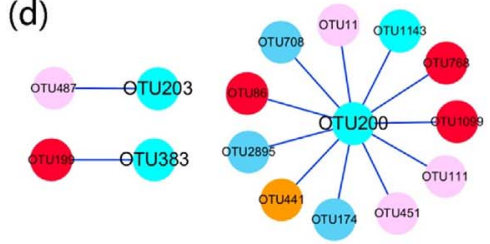

Acidobacteria

Nitrospirae (b)

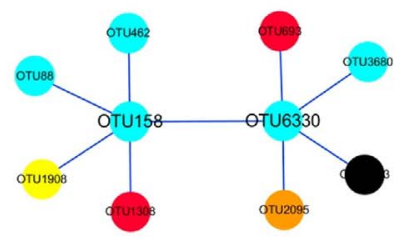

(e)

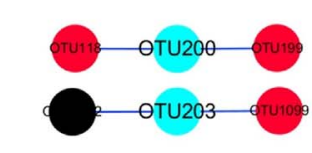

Bacteroidetes

Proteobacteria (c)

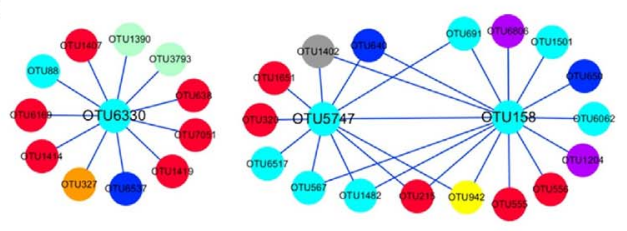

(f)

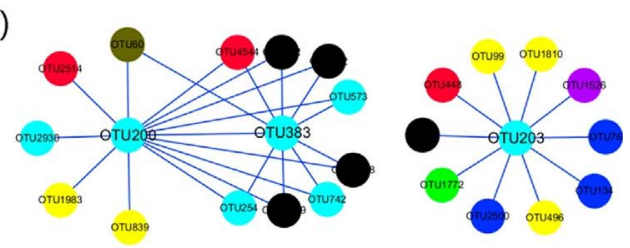

$\square$ Chloroflexi $\square$ Firmicutes
$\square$ Thaumarchaeota $\square$ Unclassified $\square$ Gemmatimonadetes

- Verrucomicrobia

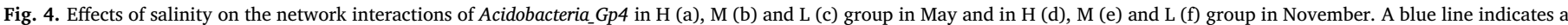

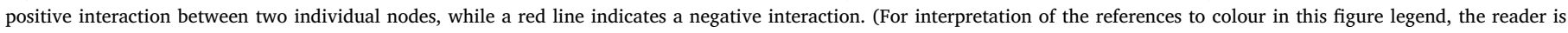
referred to the web version of this article.)

Microorganisms in naturally saline habitats are supposed to share some common strategies to resist high salinity (Ma and Gong, 2013). Multiple adaptations must be developed for maintaining resistant populations to cope with salt stress. Our pMENs analysis showed that salinity significantly altered microbial interactions. Thus, it is likely that the adjustment of microbial interactions at community level is a strategy to cope with high salinity stress. In the network, positive links could be attributed to niche overlap and cross-feeding, while negative relationships could be attributed to competition and amensalism (Faust and Raes, 2012). Microbial communities owned more positive links among OTUs in the $\mathrm{H}$ group, indicating that they may cooperate or adapt to similar ecological niches. Adversely, some microorganisms who did not own the ability to compete with others would be filtered out (Pointing et al., 2009). Specifically, the genus Flavobacterium (Bacteroidetes) is considered as moderately halophilic bacteria (Ren and Zhou, 2003). However, the relative abundance of Flavobacterium was highest in H group. Acidobacteria_Gp4 was most abundant genus of Acidobacteria phylum in M and L groups. The sub-networks of Flavobacterium/Acidobacteria_Gp4 indicated that the top three Flavobacterium OTUs in $\mathrm{H}$ group had more complex interactions than their corresponding OTUs in M and L groups, while Acidobacteria_Gp4 had much less complex interactions in $\mathrm{H}$ group. It appeared that salinity selected for Flavobacterium but against Gp4. This suggested that the interactions among different microbial taxa were substantially changed by salinity, and such impacts were different among various populations.

A previous study reports that the positive correlations percentage (a)
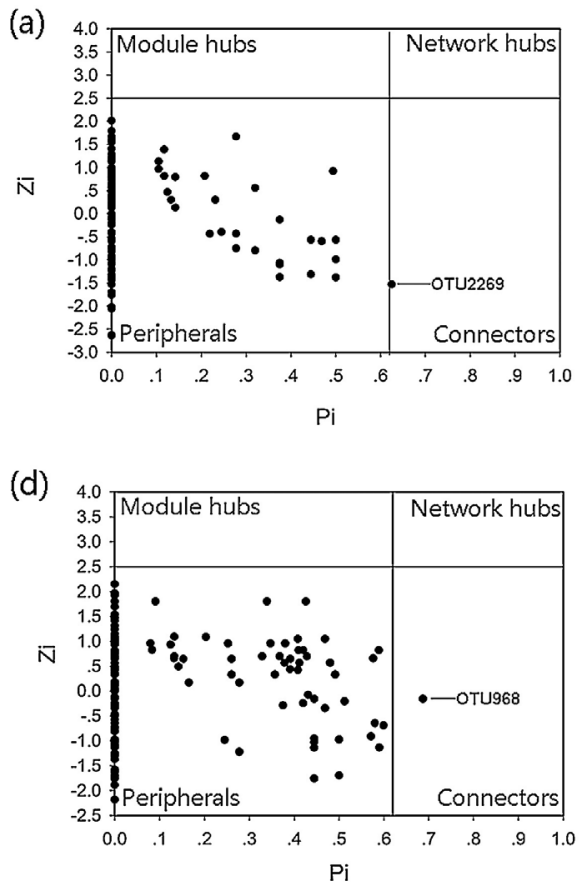

(b)
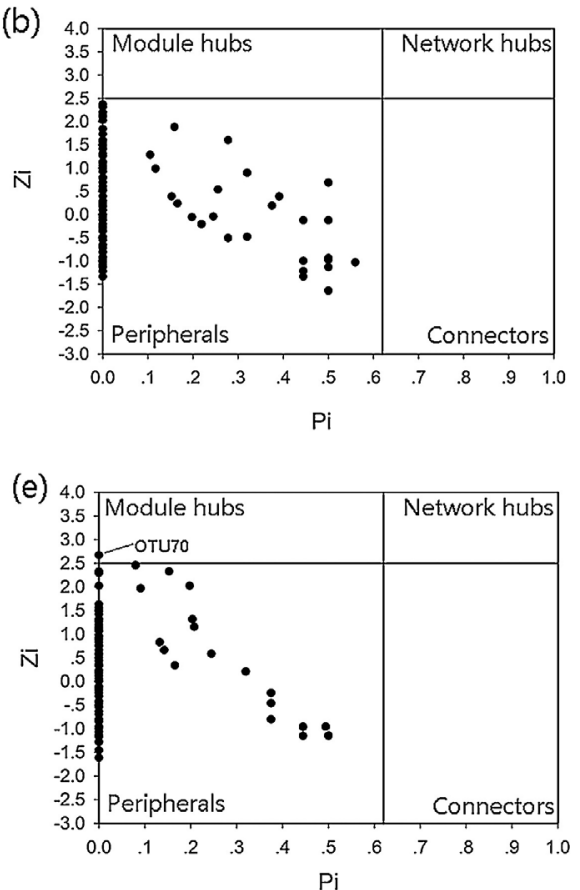

(c)

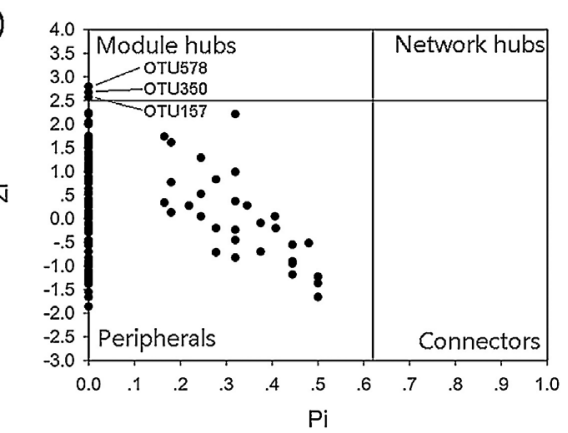

$(f)$

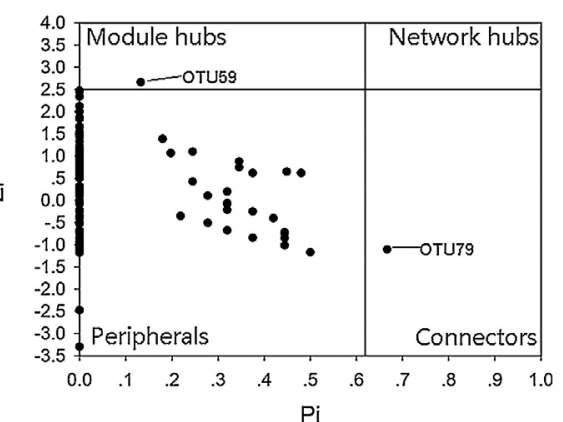

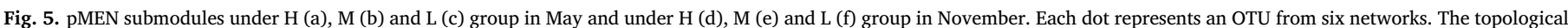

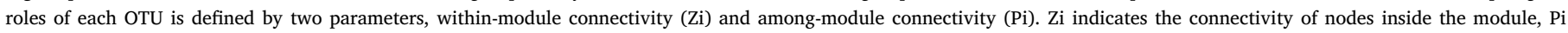
indicates the connectivity of nodes with other modules. According to values of $\mathrm{Zi} \mathrm{(2.5)}$ and Pi (0.62), the roles of nodes were classified into four categories as below. 
ranges from $57.2 \%$ to $63.4 \%$ in four $\mathrm{C}$ and $\mathrm{N}$ cycling gene networks (Sun et al., 2015a,b). It was beyond our expectation that the positive link percentage were above $98 \%$ in all the six networks. It is likely that high salt habitat forces microbes to intensify their cooperations against salt stress. Alternatively, it may be attributed to the mutualisms among microbes in long-term co-evolution processes (Zhang et al., 2014) under high salinity.

\section{Conclusions}

This study revealed the changes in the diversity, structure and interactions of soil prokaryotic communities driven by salinity. High salinity decreased prokaryote community diversity, but enriched some taxa, e.g. Proteobacteria, Bacteroidetes and Firmicutes. Additionally, high salinity enhanced microbial interactions. It implies that microbes in naturally saline habitats could develop multiple adaptations to survive under high salt concentrations, including changing the interactions among microbes. Our results provide solid evidences that microbial communities adapt to salinity through the adjustments of microbial compositions and interactions. This study is an important step towards an integrated understanding of the mechanisms that microorganisms may apply to adapt to salinity.

\section{Acknowledgements}

We gratefully acknowledge Linzhen Guo and Mengfan Yang for soil sampling and Key Laboratory of Environmental and Applied Microbiology, Chinese Academy of Sciences for 16S rRNA Miseq-sequencing analysis. This study was financially supported by the National Natural Science Foundation of China (41203001), The Innovation Team Training Plan of the Tianjin Education Committee (TD12-5037), Tianjin Municipal Science and Technology Foundation (13JCYBJC38200), Tianjin Normal University Foundation (5RL117) and China Biodiversity Observation Networks (Sino BON).

\section{Appendix A. Supplementary data}

Supplementary data associated with this article can be found, in the online version, at http://dx.doi.org/10.1016/j.apsoil.2017.08.019.

\section{References}

Barabasi, A.L., Oltvai, Z.N., 2004. Network biology: understanding the cell's functional organization. Nat. Rev. Genet. 5 (2), U101-U115.

Campbell, B.J., Kirchman, D.L., 2013. Bacterial diversity, community structure and potential growth rates along an estuarine salinity gradient. ISME J. 7 (1), 210-220.

Deng, Y., Jiang, Y.H., Yang, Y., He, Z., Luo, F., Zhou, J., 2012. Molecular ecological network analyses. mBio 13, 113.

Dong, H., Zhang, G., Jiang, H., Fields, M.W., 2006. Microbial diversity in sediments of saline Qinghai Lake, China: linking geochemical controls to microbial ecology. Microbol. Ecol. 51 (1), 65-82.

Edgar, R.C., Haas, B.J., Clemente, J.C., Quince, C., Knight, R., 2011. UCHIME improves sensitivity and speed of chimera detection. Bioinform 27 (16), 2194-2200.

Egamberdieva, D., Kamilova, F., Validov, S., Gafurova, L., Kucharova, Z., 2008. High incidence of plant growth-stimulating bacteria associated with the rhizosphere of wheat grown on salinated soil in Uzbekistan. Environ. Microbiol. 10 (1), 1-9.

Faust, K., Raes, J., 2012. Microbial interactions: from networks to models. Nat. Rev. Microbiol. 10 (8), 538-550.

Gardner, T.S., Di, B.D., Lorenz, D., Collins, J.J., 2003. Inferring genetic networks and identifying compound mode of action via expression profiling. Science 301 (5629), $102-105$.

Gerstung, M., Baudis, M., Moch, H., Beerenwinkel, N., 2009. Quantifying cancer progression with conjunctive Bayesian networks. Bioinform 25 (21), 2809-2815.

Ghollarata, M., Raiesi, F., 2007. The adverse effects of soil salinization on the growth of Trifolium alexandrinum L. and associated microbial and biochemical properties in a soil from Iran. Soil Biol. Biochem. 39 (7), 1699-1702.

Ghosh, A., Dey, N., Bera, A., Tiwari, A., Sathyaniranjan, K., Chakrabarti, K., Chattopadhyay, D., 2010. Culture independent molecular analysis of bacterial communities in the mangrove sediment of Sundarban, India. Saline Syst. 6 (1), 1.

Hidri, R., Barea, J.M., Metoui-Ben Mahmoud, O., Abdelly, C., Azcon, R., 2016. Impact of microbial inoculation on biomass accumulation by Sulla carnosa provenances, and in regulating nutrition, physiological and antioxidant activities of this species under non-saline and saline conditions. J. Plant Phys. 201, 28-41.
Horvath, S., Dong, J., 2008. Geometric interpretation of gene coexpression network analysis. PLOS Comput. Biol. 4 (8), e1000117.

Horvath, S., Zhang, B., Carlson, M., Lu, K.V., Zhu, S., Felciano, R.M., Laurance, M.F., Zhao, W., Qi, S., Chen, Z., Lee, Y., Scheck, A.C., Liau, L.M., Wu, H., Geschwind, D.H., Febbo, P.G., Kornblum, H.I., Cloughesy, T.F., Nelson, S.F., Mischel, P.S., 2006. Analysis of oncogenic signaling networks in glioblastoma identifies ASPM as a molecular target. Proc. Natl. Acad. Sci. U. S. A. 103 (46), 17402-17407.

Keshri, J., Mody, K., Jha, B., 2013. Bacterial community structure in a semi-Arid haloalkaline soil using culture independent method. Geomicrobiol. J. 30 (6), 517-529.

Killham, K., 1994. Soil Ecology. Cambridge University Press, U.K.

Langfelder, P., Horvath, S., 2007. Eigengene networks for studying the relationships between co-expression modules. BMC Syst. Biol. 1, 54.

Li, X., Rui, J., Mao, Y., Yannarell, A., Mackie, R., 2014. Dynamics of the bacterial community structure in the rhizosphere of a maize cultivar. Soil Biol. Biochem. 68, 392-401.

Lin, Q., De, V.J., Li, J., Li, X., 2016. Temperature affects microbial abundance, activity and interactions in anaerobic digestion. Bioresour. Technol. 209, 228-236.

Ma, B., Gong, J., 2013. A meta-analysis of the publicly available bacterial and archaeal sequence diversity in saline soils. World J. Microbiol. Biotechnol. 29 (12), 2325-2334.

Mavi, M.S., Marschner, P., 2012. Drying and wetting in saline and saline-sodic soils-effects on microbial activity, biomass and dissolved organic carbon. Plant Soil. 355 (1-2), 51-62.

Muhammad, S., Müller, T., Joergensen, R.G., 2006. Decomposition of pea and maize straw in Pakistani soils along a gradient of salinity. Biol. Fertil. Soils 43 (1), 93-101.

Newman, M.E.J., 2006. Finding community structure in networks using the eigenvectors of matrices. Phys. Rev. E 74 (3), 036104.

Nicholson, C., Fathepure, B., 2005. Aerobic biodegradation of benzene and toluene under hypersaline conditions at the Great Salt Plains, Oklahoma. FEMS Microbiol. Lett. 245 (2), 257-262.

Oldham, M.C., Horvath, S., Geschwind, D.H., 2006. Conservation and evolution of gene coexpression networks in human and chimpanzee brains. Proc. Natl. Acad. Sci. U. S. A. 103 (47), 17973-17978.

Olesen, J.M., Bascompte, J., Dupont, Y.L., Jordano, P., 2007. The modularity of pollination networks. Proc. Natl. Acad. Sci. U. S. A. 104 (50), 19891-19896.

Pankhurst, C., Yu, S., Hawke, B., Harch, B., 2001. Capacity of fatty acid profiles and substrate utilization patterns to describe differences in soil microbial communities associated with increased salinity or alkalinity at three locations in South Australia. Biol. Fertil. Soils 33 (3), 204-217.

Pointing, S.B., Chan, Y., Lacap, D.C., Lau, M.C.Y., Jurgens, J.A., Farrell, R.L., 2009. Highly specialized microbial diversity in hyper-arid polar desert. Proc. Natl. Acad. Sci. U. S. A. 106 (47), 19964-19969.

Qadir, M., Ghafoor, A., Murtaza, G., 2000. Amelioration strategies for saline soils: a review. Land Degrad. Dev. 11 (6), 501-521.

Ren, P., Zhou, P., 2003. Research progress of moderately halophilic bacteria. Acta Microbiol. Sin. 43 (3), 427-431 (in Chinese with English abstract).

Rousk, J., Elyaagubi, F.K., Jones, D.L., Godbold, D.L., 2011. Bacterial salt tolerance is unrelated to soil salinity across an arid agroecosystem salinity gradient. Soil Biol. Biochem. 43 (9), 1881-1887.

Rui, J., Li, J., Zhang, S., Li, X., 2015. The core populations and co-occurrence patterns of prokaryotic communities in household biogas digesters. Biotech. Biofuels 8, 158 .

Samaei, M.R., Mortazavi, S.B., Bakhshi, B., Jafari, A.J., 2013. Isolation genetic identification, and degradation characteristics of $\mathrm{n}$-hexadecane degrading bacteria from tropical areas in Iran. Fresenius Environ. Bull. 22 (4B), 1304-1312.

Sand, M., Stahl, J., Waclawska, L., Ziegler, C., Averhoff, B., 2014. Identification of an osmo-dependent and an osmo-independent choline transporter in Acinetobacter baylyi: implications in osmostress protection and metabolic adaptation. Environ. Microbiol. 16 (6), 1490-1502.

Sardinha, M., Müller, T., Schmeisky, H., Joergensen, R.G., 2003. Microbial performance in soils along a salinity gradient under acidic conditions. Appl. Soil Ecol. 23 (3), 237-244.

Sun, H., Zhao, G., Hou, Y., Liu, B., Sun, L., 2015a. Influence of alkali-saline on soil microbial diversity in Songnen grassland. Fresenius Environ. Bull. 24 (6B), 2113-2117.

Sun, X., Wang, S., Lin, Q., Zhou, J., Yang, Y., 2015b. Molecular ecological network analyses revealing the effects of livestock grazing on soil microbial community in the Tibetan grassland. Microbiol. China 42 (9), 1818-1831.

Valenzuela-Encinas, C., Neria-González, I., Alcántara-Hernández, R.J., Estrada-Alvarado, I., Dendooven, L., Marsch, R., 2009. Changes in the bacterial populations of the highly alkaline saline soil of the former lake Texcoco (Mexico) following flooding. Extremophiles 13 (4), 609-621.

Wang, Q., Garrity, G.M., Tiedje, J.M., Cole, J.R., 2007. Naïve Bayesian classifier for rapid assignment of rRNA sequences into the new bacteria taxonomy. Appl. Environ. Microbiol. 73 (16), 5261-5267.

Wang, M., Li, J., Yue, F., Zhu, Z., Liu, X., Guo, X., 2011. Changes of soil salinity in saline soil of Tainjin. Chin. J. Ecol. 30 (9), 1949-1954 (in Chinese with English abstract).

Wang, Y., Zhang, R., Zheng, Q., Deng, Y., Nostrand, J.D.V., Zhou, J., Jiao, N., 2015. Bacterioplankton community resilience to ocean acidification: evidence from microbial network analysis. ICES J. Mar. Sci. 73 (3), 865-875.

Yang, H., Hu, J., Long, X., Liu, Z., Rengel, Z., 2016. Salinity altered root distribution and increased diversity of bacterial communities in the rhizosphere soil of Jerusalem artichoke. Sci. Rep. 6, 20687.

Yeung, M.K.S., Tegner, J., Collins, J.J., 2002. Reverse engineering gene networks using singular value decomposition and robust regression. Proc. Natl. Acad. Sci. U. S. A. 99 (9), 6163-6168.

Yin, H., Niu, J., Ren, Y., Cong, J., Zhang, X., Fan, F., Xiao, Y., Zhang, X., Deng, J., Xie, M., He, Z., Zhou, J., Liang, Y., Liu, X., 2015. An integrated insight into the response of 
sedimentary microbial communities to heavy metal contamination. Sci. Rep. 5, 14266.

Yue, F., Liu, X., Li, J., Zhu, Z., Wang, Z., 2010. Using nitrogen isotopic approach to identify nitrate sources in waters of Tianjin, China. Bull. Environ. Contam. Toxicol. 85 (6), 562-567.

Zhang, B., Horvath, S., 2005. A general framework for weighted gene co-expression network analysis. Stat. Appl. Genet. Mol. Biol. 4, 17.

Zhang, Y., Zhao, Z., Dai, M., Jiao, N., Herndl, G.J., 2014. Drivers shaping the diversity and biogeography of total and active bacterial communities in the South China Sea. Mol. Ecol. 23 (9), 2260-2274.

Zhou, J., Deng, Y., Luo, F., He, Z., Tu, Q., Zhi, X., 2010. Functional molecular ecologica networks. mBio 1 (4), e00169-10.

Zhou, J., Deng, Y., Luo, F., He, Z., Yang, Y., 2011. Phylogenetic molecular ecological network of soil microbial communities in response to elevated CO2. mBio 2 (4), e00122-11. 